\title{
Control of peripheral sympathetic prevertebral ganglion neurones by colonic mechanosensory afferents
}

\author{
S M Miller
}

Sympathetic innervation of the gastrointestinal tract arises from abdominal prevertebral ganglia (PVG) comprising the coeliac, superior mesenteric, and inferior mesenteric ganglia. These ganglia are integrating centres between the central nervous system and enteric nervous system and their output regulates intestinal motility, intestinal blood flow, and water and electrolyte secretion. ${ }^{1}$ Much of the synaptic input to PVG neurones arises from cholinergic mechanosensory neurones whose cell bodies lie in the gut wall and provide the PVG with information about the mechanical state of the gut. The mechanosensory ("intestinofugal") neurones and their axons constitute the afferent limb of a peripheral reflex between enteric and sympathetic PVG neurones. The efferent limb of the reflex consists of the PVG neurones and their axons, which carry inhibitory sympathetic output back to the gut. ${ }^{2}$ Experiments were performed in vitro to examine the peripheral reflex loop between colonic enteric neurones and sympathetic superior mesenteric ganglion (SMG) neurones in mouse. Preparations consisted of the SMG attached via intermesenteric and colonic nerves to a segment of distal colon. ${ }^{3}$ The distal end of the colonic segment was attached to a fluid filled reservoir. With this set up, the segment could empty its contents into the reservoir during a contraction and then refill during relaxation. Reflex activity was monitored intracellularly from SMG neurones while simultaneously recording intraluminal volume and muscle wall tension from the colonic segments. ${ }^{4}$ The results showed that colonic mechanosensory input to the SMG increased when the colon distended during filling, decreased and was absent when colonic contraction emptied the segment, and increased again when the colon relaxed and refilled (fig 1). These results suggested that the colonic mechanosensory afferent neurones monitored changes in intracolonic volume. To test this hypothesis, the colon segment was manipulated to prevent it from emptying during contraction or refilling after contraction. When the colon segment was prevented from emptying, the mechanosensory afferent input to the SMG neurones was not diminished during contraction. Conversely, if the colon segment could not refill after emptying, mechanosensory input to the SMG was absent until the moment when the colon was permitted to refill. To examine whether colonic mechanosensory afferents are activated by distension of the longitudinal, circular, or both muscle layers, the colon segment was made

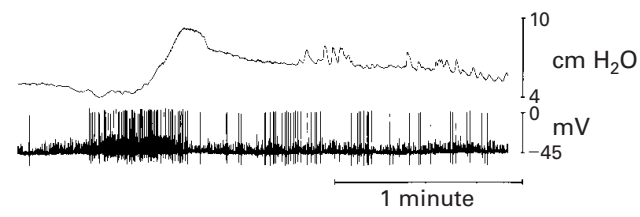

Figure 1 Changes in excitatory colonic mechanosensory synaptic input (lower trace) to a mouse superior mesenteric ganglion neurone during spontaneous change in intracolonic pressure and volume (upper trace)

into a flat sheet and the sheet was stretched in the direction of the longitudinal or circular muscle axis while simultaneously recording electrical activity of SMG neurones. ${ }^{5}$ The results showed that the mechanosensory afferent pathway was activated when the colon was stretched in the circular muscle but not in the longitudinal muscle axis, suggesting that the mechanosensory endings in the colon wall are functionally arranged with the circular muscle layer. Retrograde labelling techniques were used to identify the cell bodies and processes of the colonic intestinofugal neurones. ${ }^{6}$ Their cell bodies were found in the myenteric ganglia, usually only one, but sometimes two labelled neurones per myenteric ganglion. Confocal microscopy will be used in future studies to locate their mechanosensory endings in the colon wall.

Recent studies have identified two messenger molecules which may mediate afferent input to PVG and modulate sympathetic efferent output, and thus may be potential targets for therapy. The first is carbon monoxide (CO), a gaseous molecule suggested to be an important signalling molecule in the body. ${ }^{7}$ Gastrointestinal transit is markedly altered in mice lacking haeme oxygenase 2 (HO-2), the biosynthetic enzyme for $\mathrm{CO}$ biosynthesis in the nervous system, suggesting that $\mathrm{CO}$ may play a role in regulating the gut. ${ }^{8}$ Immunohistochemistry using two different polyclonal antibodies raised against $\mathrm{HO}-2$ was performed to examine the distribution of $\mathrm{CO}$ producing enzyme in the extrinsic and intrinsic nerves to the gastrointestinal tract. Both antibodies produced the same result, that HO-2 immunoreactivity was found in enteric neurones, interstitial cells of Cajal, and in sympathetic PVG neurones, suggesting that these cells may be important sources of $\mathrm{CO}$ regulating the gastrointestinal tract. ${ }^{9}$ Development of specific pharmacologi-

Abbreviations used in this paper: $\mathrm{PVG}$, prevertebral ganglia; SMG, superior mesenteric ganglion; $\mathrm{CO}$, carbon monoxide; $\mathrm{HO}-2$, haeme oxygenase 2 ; LR-like IR, leptin receptor-like immunoreactivity. 


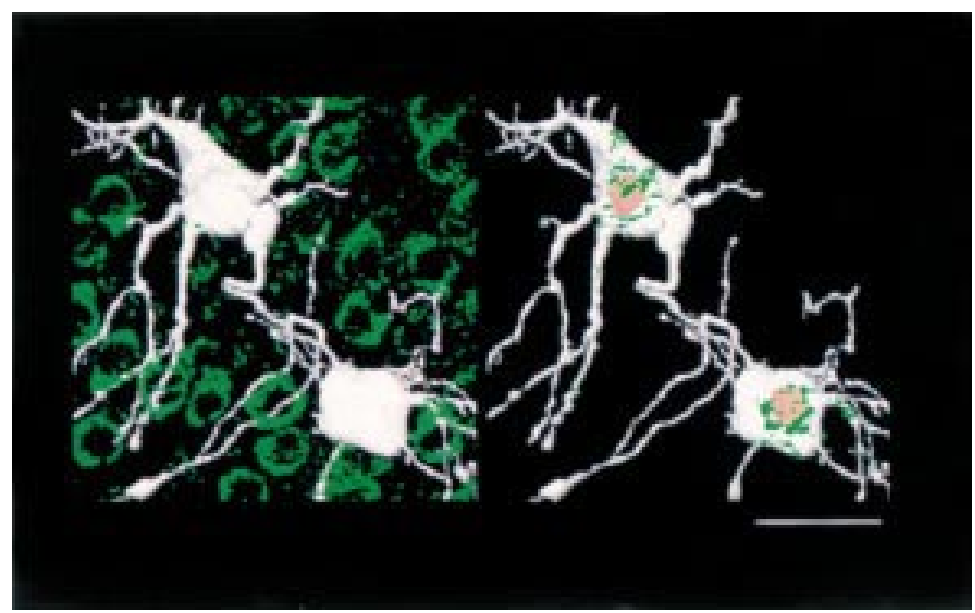

Figure 2 Three dimensional reconstructed images of mouse superior mesenteric ganglion (SMG) neurones injected with lucifer yellow (LY) fluorescent dye and then immunostained for leptin receptors (LR) Left: LY filled neurones (white), LR (green). Right: LY filled neurones were made transparent revealing the intracellular distribution of LRs (green) and nuclei (pink).
LR-like IR was located intracellularly and was absent from the neuronal plasma membrane (fig 2). Double immunolabelling of PVG neurones showed almost complete colocalisation of LR-like IR and Golgi body immunoreactivity. The intracellular location of leptin receptors in sympathetic neurones is puzzling as it is unclear how leptin (which is too large to cross the plasma membrane freely) might interact with its intracellular receptor. It is possible that translocation of the leptin receptor from the Golgi body to the plasma membrane might occur under conditions when leptin levels are abnormally high (for example, in obese and diabetic animals) or abnormally low (for example, in lean or starved animals). Studies are now being undertaken to examine the distribution of leptin receptors in PVG and enteric ganglia from obese and starved animals.

Supported by NIH grant DK17632 to J Szurszewski.

1 Furness JB, Costa M. The enteric nervous system. London: Churchill Livingstone, 1987.

cal inhibitors of HO-2 is necessary for continued progress in understanding how and where $\mathrm{CO}$ can modify gastrointestinal function.

A second important messenger molecule may be the hormone leptin, a protein produced by adipocytes, that acts on neurones in the hypothalamus to control food intake and energy balance. ${ }^{10}$ Immunohistochemistry with an antibody raised against the leptin receptor showed that sympathetic PVG and enteric ganglia of the stomach, small intestine, and colon of normally fed mice and rats contained abundant leptin receptor-like immunoreactivity (LR-like IR). These results suggest that peripheral autonomic neurones, such as those in feeding centres of the brain, may be involved in the action of leptin on regulating food intake and metabolism. Confocal microscopy and three dimensional reconstruction of dye filled and immunolabelled PVG neurones were used to examine the distribution of leptin receptors in PVG neurones. ${ }^{11}$ The results showed that
2 Szurszewski JH, Miller SM. Physiology of prevertebral ganglia. In: Johnson LR, ed. Physiology of the gastrointestinal tract, 3rd edn. New York: Raven Press, 1987:795-877.

3 Miller SM, Szurszewski JH. Colonic mechanosensory afferent input to neurons in the mouse superior mesenteric ganglion. Am F Physiol 1997;272:G357-66.

4 Miller SM, Szurszewski JH. Colonic mechanoreceptors projecting to mouse prevertebral ganglia function as volume receptors. $\mathcal{F}$ Gastrointest Motil 1989;1:A79.

5 Miller SM, Szurszewski JH. Colonic mechanosensory afferents projecting to the mouse superior mesenteric ganglion are in parallel tension receptors located in the circular are in parallel tension receptors located in
muscle layer. Gastroenterolgy 1990;99:A1222.

6 Miller SM, Ermilov LG, Hanani M, et al. The neuritic procMiller SM, Ermilov LG, Hanani M, et al. The neuritic proc-
esses of Dogiel type II colonofugal neurons are arranged esses of Dogiel type II colonofugal neurons are arranged
parallel to the circular muscle layer of the mouse colon. parallel to the circular muscle

7 Maines MD. The heme oxygenase system: a regulator of second messenger gases. Ann Rev Pharmacol Toxicol 1997;37:517-54

8 Zakhary R, Poss KD, Jaffrey SR, et al. Targeted gene deletion of heme oxygenase 2 reveals neural role for carbon monoxide. Proc Natl Acad Sci USA 1997;94:14848-53.

9 Miller SM, Farrugia G, Schmalz PF, et al. Heme oxygenase 2 is present in interstitial cell networks of the mouse small intestine. Gastroenterology 1998;114:239-44. molecular targets for obesity treatment. Science 1998;280: 1383-7.

11 Miller SM, Schmalz PF, Benarroch E, et al. Leptin receptor immunoreactivity in sympathetic prevertebral ganglion neurons of mouse and rat. Neurosci Lett 1999;265:75-8.
10 Camfield LA, Smith FJ, Burn P. Strategies and potential 